\title{
Preliminary Evidence for the Emergence of a Health Care Online Community of Practice: Using a Netnographic Framework for Twitter Hashtag Analytics
}

Damian Roland $^{1 *}$, BMedSci, BMBS, PhD; Jesse Spurr $^{2 *}$, BN; Daniel Cabrera ${ }^{3 *}$, MD

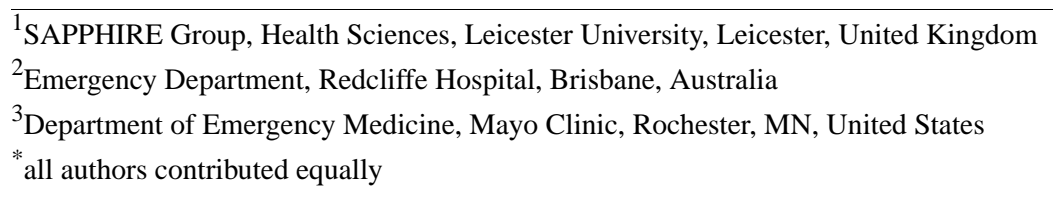

Corresponding Author:

Damian Roland, BMedSci, BMBS, PhD

SAPPHIRE Group

Health Sciences

Leicester University

Centre for Medicine

Leicester, LE1 7RH

United Kingdom

Phone: 447727158213

Fax: 441162586089

Email: dr98@le.ac.uk

\section{Abstract}

Background: Online communities of practice (oCoPs) may emerge from interactions on social media. These communities offer an open digital space and flat role hierarchy for information sharing and provide a strong group identity, rapid flow of information, content curation, and knowledge translation. To date, there is only a small body of evidence in medicine or health care to verify the existence of an oCoP.

Objective: We aimed to examine the emergence of an oCoP through the study of social media interactions of the free open access medical education (FOAM) movement.

Methods: We examined social media activity in Twitter by analyzing the network centrality metrics of tweets with the \#FOAMed hashtag and compared them with previously validated criteria of a community of practice (CoP).

Results: The centrality analytics of the FOAM community showed concordance with aspects of a general CoP (in terms of community, domain, and practice), as well as some specific traits of a health care community, including social control, common purpose, flat hierarchy, and network-based and concrete achievement.

Conclusions: This study demonstrated preliminary evidence of an oCoP focused on education and based on social media interactions. Further examination of the topology of the network is needed to definitely prove the existence of an oCoP. Given that these communities result in significant knowledge translation and practice change, further research in this area appears warranted.

(J Med Internet Res 2017;19(7):e252) doi: 10.2196/jmir.7072

\section{KEYWORDS}

social media; network; community networks; community of practice; \#FOAMed; Twitter

\section{Introduction}

Creation, dissemination, and management of knowledge are cornerstones of safe and effective health care, but achieving these goals in functional and successful large care systems remains enormously challenging. Within this construct of health systems [1], the concept of a network is vital for improving patient care by fostering collaboration, stimulating engagement and knowledge acquisition and management, and promoting learning [2]. Recently, social media-based platforms have been 
proffered as educational tools [3,4], based on the critical assumption that activity in online networks can lead to the emergence of personal learning networks [5] and communities of practice (CoPs) [6].

CoPs are an anthropological concept, defined as a group of people who organize around a specific component of knowledge (eg, a profession or particular task). They create, organize, and share information and not only develop specific domains but also foster development of individual members [6]. These communities are organized around a domain, community, and practice, where the domain is a realm of knowledge that the members of the group have interest in and value as important; the community is the coalescence of interactions and negotiations between members around the domain; and practice is the results of the cooperation leading to the creation of tangible resources affecting their practice of the knowledge. The presumed natural evolution of organically organized online communities [7] is the description and identification of an online CoP (oCoP) [8]. This construct offers an enormous potential for the creation, dissemination, and management of knowledge and people using a relatively minimal amount of resources and appearing as an ideal framework for information-oriented teams. These have arisen from the Internet in general and particularly in social media platforms [9] because of their ability to efficiently connect and engage groups, provide peer-based education [10], and manage meaningful knowledge [8].

The concept of CoP has been thoroughly described in health care [11], and the critical components of an appropriate network were refined and expanded by Aveling et al in 2012 [12]. A health care $\mathrm{CoP}$ is defined by 8 core characteristics (described below) that are themselves derived from Wenger's [6] pivotal components of community, domain, and practice.

Although CoPs are not a new phenomenon, social media platforms have enabled them to form rapidly and across international boundaries, with nonlinearity and on a large scale [13]. These new oCoPs create discrete and quantifiable data flow among users (nodes) of the network. Knowledge and relationships develop by these interactions (links), with functional communities arising as information transfer increases. These communities of online learning and practice offer an open digital space for information sharing, with a flat role hierarchy, strong group identity, high engagement, and rapid flow of information, content curation, and knowledge translation. In many ways, their topology represents the structure of scale-free networks $[14,15]$. The use of social media platforms as an educational medium is dependent on these oCoPs providing reliable and manageable information for their participants [16].

Despite the recognition of several possible candidates for CoPs and their derivatives in online platforms [8], particularly in the health care sector [11] and for peer-to-peer patient interactions [17], medical learning and practice oCoPs have been rarely described in the medical literature to date. An international movement that began within the past 5 years now aims to collaborate to create, curate, and disseminate medical knowledge with the intention of changing patterns of care; an explicit aim is to reduce the knowledge translation gap [2,15]. This movement describes the concept as "free open access medical education" (FOAM) [18,19] and organizes around the Twitter hashtag \#FOAMed; the group name is synonymous with and represents its core philosophy.

The strength of oCoPs can be established if these communities are identified and their relationships described; social media education could then be validated as a viable paradigm for knowledge translation [16] that affects health care practice. The aim of this study was to show preliminary evidence of the emergence of a health care oCoP focused on knowledge translation and organized as a network architecture around the Twitter hashtag \#FOAMed. By using data extracted from our \#FOAMed hashtag analysis, we aimed to demonstrate that the FOAM community fulfills the core characteristics of a CoP, as described by Wenger [6] and expanded by Aveling et al [12], and is a community of individuals with different roles who belong to different organizations and places, who organize themselves around a concrete domain and specific goal, and who function to support and promote each other's development to achieve change.

We hypothesized that the FOAM community, defined by the explicit interactions that include the \#FOAMed hashtag, constitutes an oCoP. To confirm this, we believed it should be possible to recognize the emergence of the community, domain, and practice of the network, while describing information flowing between nodes and the semantic relationships between the members. Describing the structure of the network, as well as the influences of nodes outside the network (eg, spambots), requires a robust intrinsic familiarity with the community; therefore, we approached the analysis using a netnographic methodology [20,21] based on our personal participation in the FOAM movement.

\section{Methods}

The challenge of analyzing billions of interactions in social media [22,23] is a relatively recent area of activity that requires a high level of expertise and computational power. We created a Symplur Signals database (Symplur LLC, Upland, CA, USA) around the Twitter hashtag \#FOAMed and interrogated it from March 1, 2013, through August 31, 2015; this dataset contained almost all data available for the hashtag because the accepted inception time for \#FOAMed was March 2013 and the activity occurred explicitly around the hashtag [18]. The computational tools implemented by Symplur Signals have been validated previously for the analysis of Tweet chats [24]. There is a large amount of FOAM community activity, such as blogs, podcasts, and conference proceedings, that is not directly related to the \#FOAMed hashtag or Twitter; however, these products are typically references in community activity and it is highly unlikely that such activity outside of Twitter would nullify the existence of the oCoP. 
Table 1. Aveling's core components of clinical communities of practice and their relationship with Wenger's classic definition.

\begin{tabular}{llll}
\hline $\begin{array}{l}\text { Composite } \\
\text { postulate } \\
\text { notation }\end{array}$ & Aveling core components of clinical communities ${ }^{\mathrm{a}}$ & \#FOAMed proof & Wenger \\
\hline A1C & Consists of interdependent groups and individuals & $\begin{array}{l}\text { The \#FOAMed hashtag connects individuals who demon- } \\
\text { strate interactions with each other. Over time, influencers } \\
\text { increase in number and become divergent rather than con- } \\
\text { vergent. }\end{array}$ & Commity \\
& &
\end{tabular}

A2C Consists of members who may cross clinical and organizational boundaries

A3D Consists of members united by a common purpose of bridging the gap between best scientific evidence and current clinical practice

A4CDP Consists of members who come together not only to learn or share knowledge but to achieve those aims

A5DCP Exploits the networks' inherent potential for effective and low-cost knowledge generation and diffusion

Operates through both vertical and lateral structures control mechanisms to achieve change

$\mathrm{A} 8 \mathrm{P}$

Harnesses the power of the community and its collective wisdom when seeking solutions to problems; includes contextual factors and local solutions
Discussion around \#FOAMed results in positive attributions regarding content.

\#FOAMed generates subnetworks around individuals or content nodes (such as specific websites).

The network expands through increasing individuals who influence others across increasingly wide geographic areas.

Key nodes exert influence, but this changes through time.

\#FOAMed hashtag is used by a variety of individuals and organizations. Over time, geographic area of use increases.

Content of \#FOAMed remains around health care-related Domain themes, centered on creation and access to content.

Community, domain, practice

Domain, community, practice

Community

Community, practice

Interactions (measured through mentions) expand rather Practice than contract over time.

\footnotetext{
${ }^{\mathrm{a}}$ Adapted from Aveling et al [12]. Used with permission.

${ }^{\mathrm{b}}$ Data from Wenger [6].
}

To reduce potential bias in the analysis, we submitted a protocol to the Symplur Signals team defining the a priori analytic strategy. Within this protocol, we proposed several proofs to demonstrate concordance with the Aveling-Wenger postulate for a CoP. Table 1 lists these proofs, matched to the 8 distinct core characteristics. For example, evidence of interdependent groups and individuals (A1C) would be demonstrated by showing that the \#FOAMed hashtag was used by individuals who had interactions with each other and that, over time, influencers increased in number, becoming divergent rather than convergent. To do this, we would therefore need to describe \#FOAMed hashtag use quantitatively. Conceptually, we aimed to analyze \#FOAMed hashtag activity as a network. The hashtag [25] constitutes the core scaffold in which the identified social activity occurs. The network consists of Twitter users (nodes), and interactions (links) with specific metrics of interactions such as retweets, favorites, and engagements, which constitute the connections and distribution by which networks are typically constructed [26,27].

Given the social nature of the Twitter platform, we used the social network analysis component of the netnographic framework for analysis, as described by Kozinets [20]; this method is particularly effective at identifying external influences that are not meaningful to the community (eg, spambots, large commercial interests), as well identifying digital artefacts that are created by mistake or are otherwise inappropriate (eg, links to unrelated events associated with the hashtag but used by a completely different community). From a netnographic perspective, it is difficult to appreciate the metrics of centrality without a personal understanding of the symbolism, meaning, and consumer patterns of the groups [28]; however, as members of the community of study, we had an immersive and descriptive understanding of the FOAM movement. Our intimate understanding allowed us to determine (via a consensus view of the authors) the relevance of the top 100 shared links identified in the ethnographic analysis of the FOAM community We generally excluded broken links, links to landing pages of websites or home pages, and any other links that did not point to a webpage with a publicly visible forum for comments and feedback or had no comments posted. This study was deemed exempt from ethics board review by the English National Health Service Health Research Authority.

\section{Results}

During the study period, we identified 49,459 active users who issued 429,606 tweets and created 1,258,692,900 impressions (ie, the number of times a user is served a Tweet in their timeline or search results); this translated to more than 8 tweets and 25,000 impressions per user involved in the conversation (Table 2 ). The user and hashtag activity, expressed as number of daily tweets, increased during the study period (from $\sim 250$ to $\sim 700$ tweets/day) (Figure 1). User participation increased substantially during the same time frame, rising from around 1000 users to more than 45,000; an additional 1000 users per month have participated since March 2014 (Figure 2). Multimedia Appendix 1 shows the top influencers. In terms of engagement, 27,635 
users $(55.71 \%)$ participated with 1 tweet (representing $6.43 \%$ of all tweets), while 2603 users (5.25\%) tweeted more than 20 times and were responsible for $72.45 \%$ of all tweets (Table 3 ).

From a network distribution perspective, the top 200 users created 148,185 tweets, representing $34.49 \%$ of all tweets of the community. The Symplur Signals analytics algorithm created a centrality map by considering nodes (users) and their interactions (links) to assign weight and used the metrics of mentions, authority, and hub to assign the weight of centrality $[29,30]$. The 100 users with the highest weights also tended to have high authority and edge scores (Multimedia Appendix 2), and from a netnographic perspective, they were recognizable as community leaders. Figure 3 outlines their status in relation to the number of interactions with other participants in a graphical representation, where the size of the node represents its network centrality (relative influence) and the links depict the strength and directions of the interactions between members (nodes) of the network. Also, Figure 4 depicts a perspective of the communication between nodes on a conversation matrix showing the frequency and strength of interactions among the most active community members.

Table 2. Metrics of the Twitter hashtag \#FOAMed ${ }^{\mathrm{a}}$.

\begin{tabular}{|c|c|c|c|c|c|}
\hline Metric & Total & No. per month & No. per week & No. per day & No. per hour \\
\hline Tweets & 429,606 & 14,132 & 3298 & 471 & 20 \\
\hline Users who tweeted & 49,459 & 1627 & 380 & 54 & 2 \\
\hline Tweets per user & 8.69 & 0.29 & 0.07 & $9.52 \times 10^{-3}$ & $3.97 \times 10^{-4}$ \\
\hline Impressions & $1.26 \times 10^{9}$ & $4.14 \times 10^{7}$ & $9.66 \times 10^{6}$ & $1.38 \times 10^{6}$ & $5.75 \times 10^{4}$ \\
\hline Impressions per user & 25,447 & 837 & 195 & 28 & 1 \\
\hline
\end{tabular}

${ }^{\mathrm{a}}$ Hashtag activity was monitored from March 1, 2013, through August 31, 2015.

Figure 1. Tweet activity (number of tweets per day) using the \#FOAMed hashtag from March 1, 2013, through August 31, 2015.

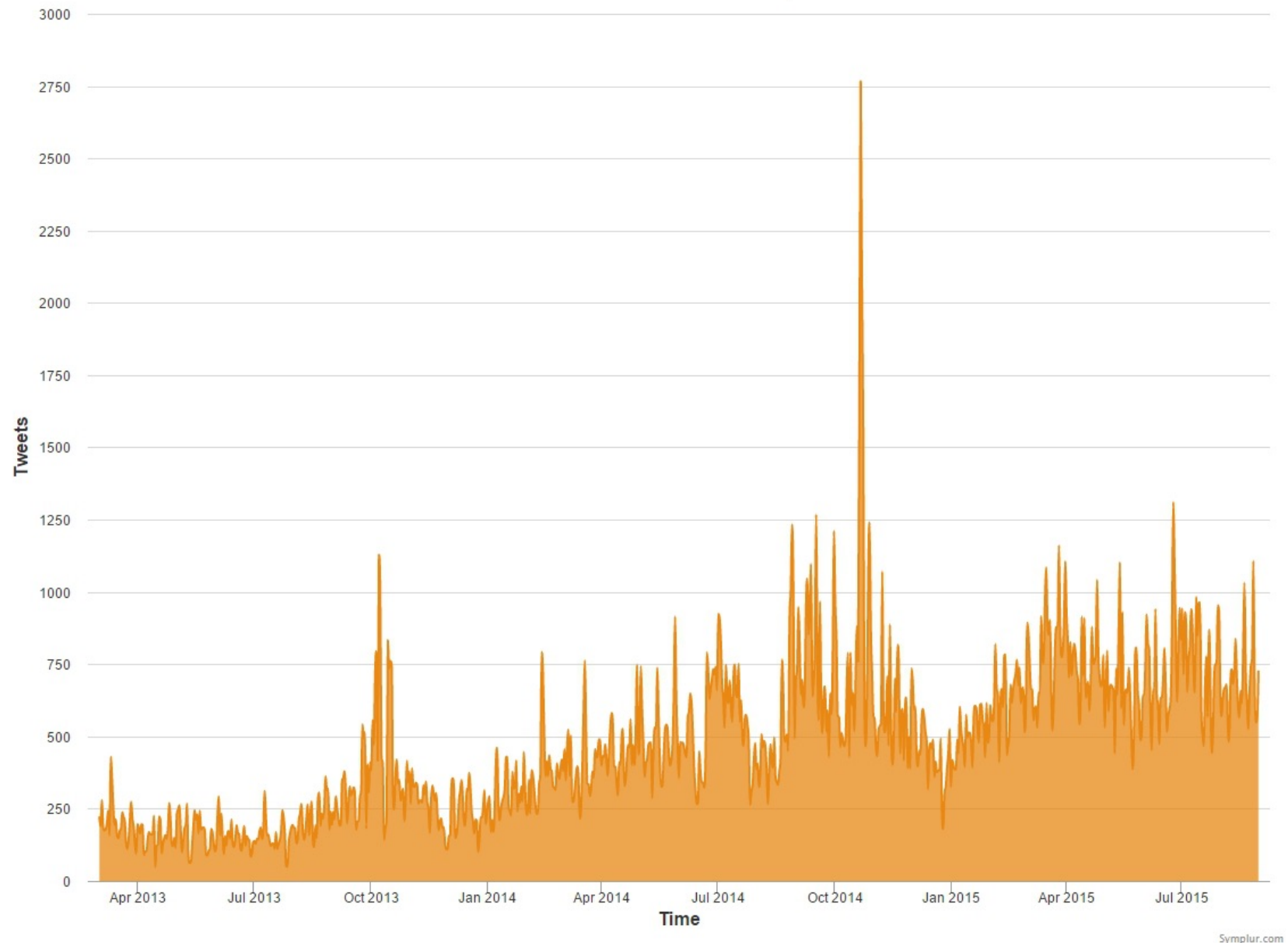


Figure 2. Cumulative number of users participating in the \#FOAMed community.

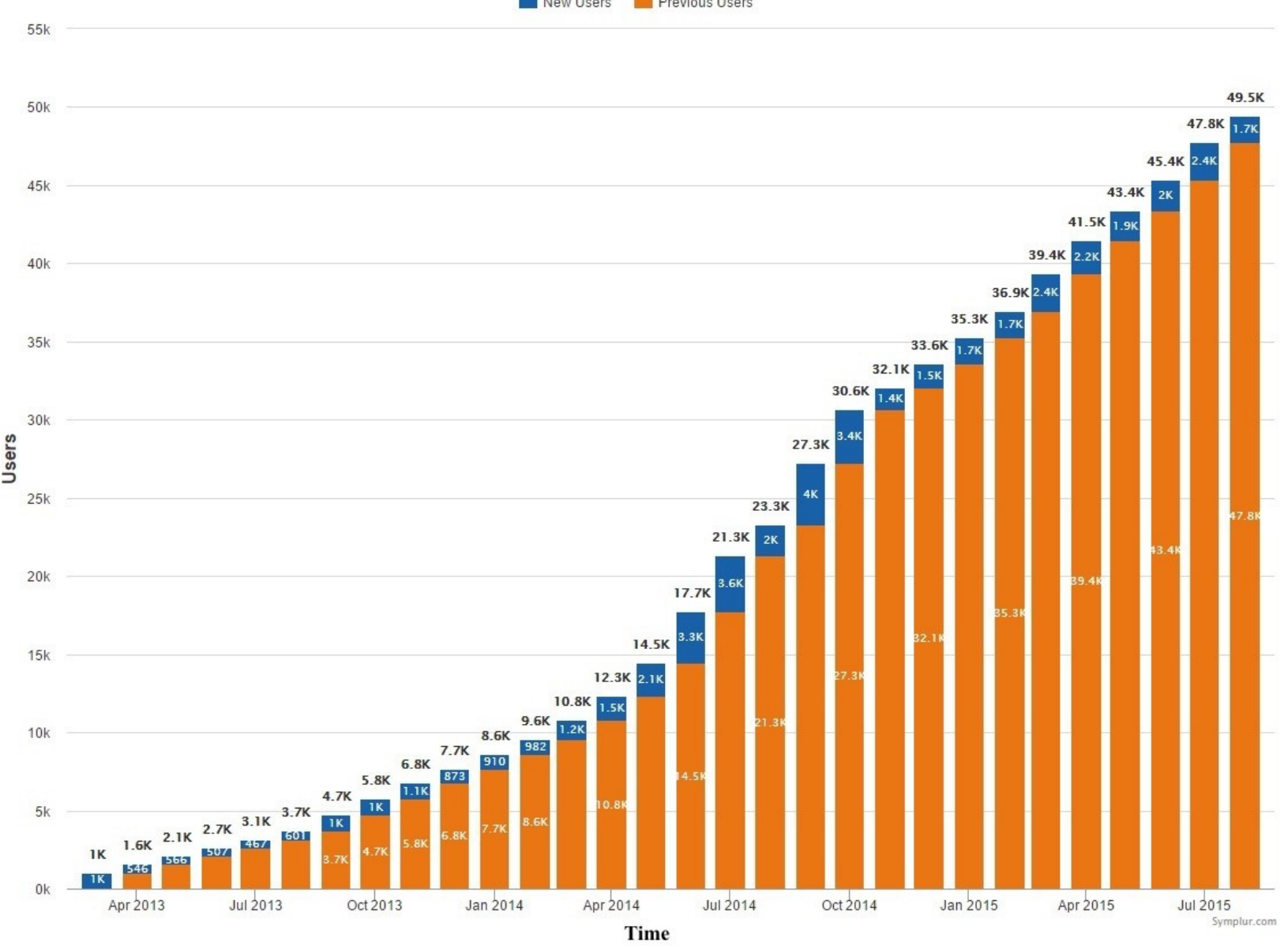


Table 3. Twitter engagement metrics for the \#FOAMed hashtag ${ }^{\mathrm{a}}$.

\begin{tabular}{|c|c|c|}
\hline User activity by no. of tweets & Total users, $\mathrm{n}(\%)$ & Proportion of all tweets, $\%$ \\
\hline 1 & $27,635(55.71)$ & 6.43 \\
\hline 2 & $7078(14.27)$ & 3.30 \\
\hline 3 & $3703(7.46)$ & 2.59 \\
\hline 4 & $2103(4.24)$ & 1.96 \\
\hline 5 & $1366(2.75)$ & 1.59 \\
\hline 6 & $1011(2.04)$ & 1.41 \\
\hline 7 & $698(1.41)$ & 1.14 \\
\hline 8 & $602(1.21)$ & 1.12 \\
\hline 9 & $454(0.92)$ & 0.95 \\
\hline 10 & $382(0.77)$ & 0.89 \\
\hline 11 & $326(0.66)$ & 0.83 \\
\hline 12 & $269(0.54)$ & 0.75 \\
\hline 13 & $229(0.46)$ & 0.69 \\
\hline 14 & $182(0.37)$ & 0.59 \\
\hline 15 & $164(0.33)$ & 0.57 \\
\hline 16 & $164(0.33)$ & 0.61 \\
\hline 17 & $145(0.29)$ & 0.57 \\
\hline 18 & $122(0.25)$ & 0.51 \\
\hline 19 & $118(0.24)$ & 0.52 \\
\hline 20 & $110(0.22)$ & 0.51 \\
\hline$>20$ & $2603(5.25)$ & 72.45 \\
\hline
\end{tabular}

${ }^{\mathrm{a}}$ Total tweets, 429,606; total number of users who tweeted, 49,459. 
Figure 3. Partial graphic depiction of the centrality metrics of the top 100 \#FOAMed users based on weight (defined by mentions, hub, and authority quotients).

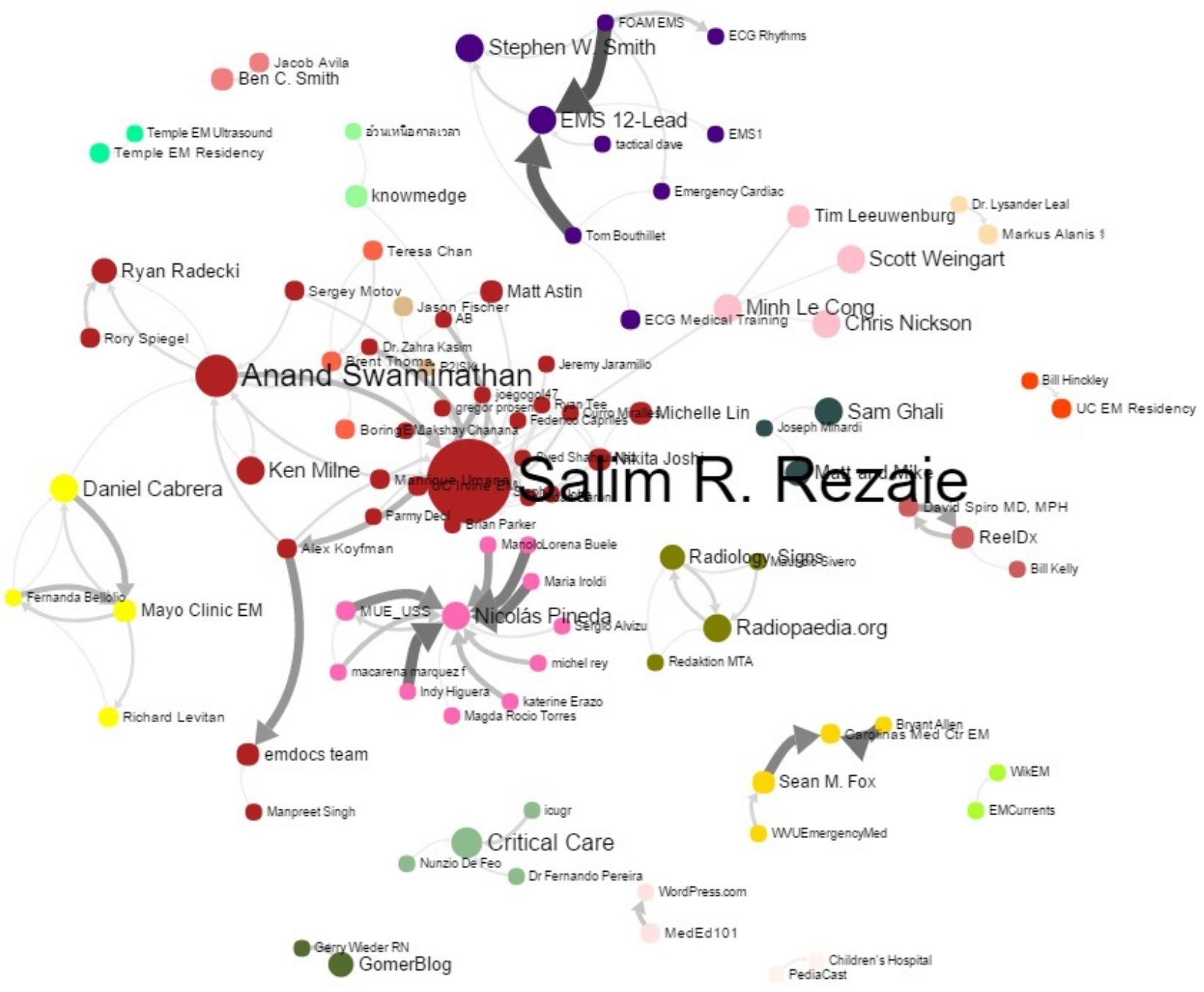

Wessexthe Bottom Line 
Figure 4. Conversation identifier depicting frequency and strength of interaction among top members of the \#FOAMed community.

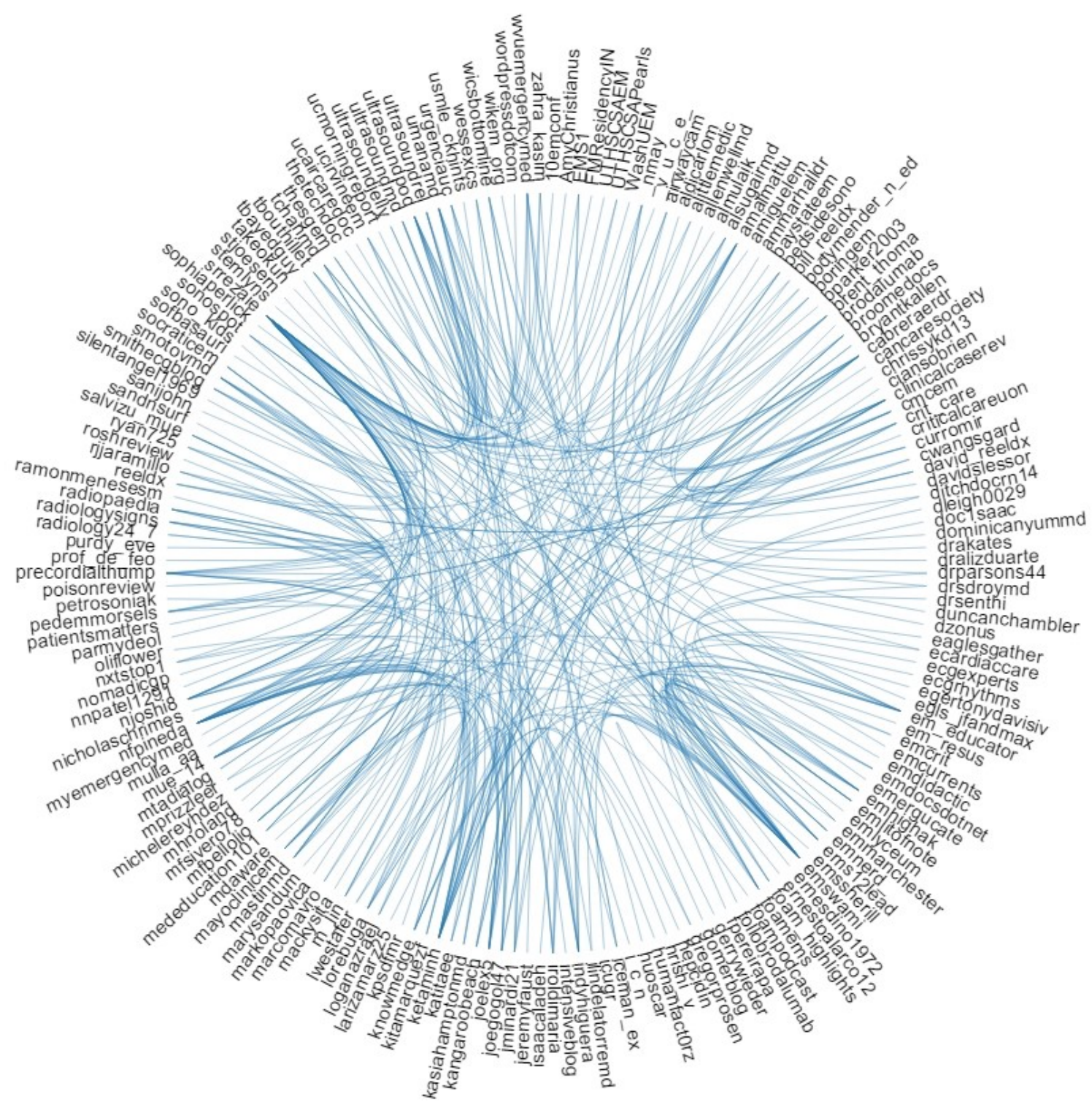

Table 4. Geographic location of \#FOAMed community members $(\mathrm{n}=9502)^{\mathrm{a}}$.

\begin{tabular}{ll}
\hline Country & $\mathrm{n}(\%)$ \\
\hline United States & $4137(43.5)$ \\
United Kingdom & $1926(20.3)$ \\
Australia & $820(8.6)$ \\
Canada & $797(8.4)$ \\
Spain & $434(4.6)$ \\
Russia & $384(4.0)$ \\
Brazil & $270(2.8)$ \\
Mexico & $270(2.8)$ \\
India & $264(2.8)$ \\
Saudi Arabia & $200(2.1)$ \\
\hline
\end{tabular}

${ }^{\mathrm{a}}$ Not all users self-identified their locations. 
In terms of geographic distribution, the community members generally lived in anglophone Western countries, although some of them were located in Latin American and Asian countries (Table 4). However, the analytic tool was unable to consider the geographic location of more than $50 \%$ of users because they did not provide self-identified geolocation information.

Multimedia Appendix 3 details the top 150 conversations (defined by Symplur in terms of level of engagement) generated in the community during the study period. The users involved were those with the highest network weight and hub index. The conversations showed a relatively low level of branching, with most having 2 or 3 levels of interactions (replies). An automatic sentiment analysis [31] showed a 0.367 positive sentiment embedded in the tweets. In terms of knowledge sharing, an analysis of the top 100 links (Multimedia Appendix 4) shared by the group shows a mix of self-created content (blogs), referral to social platforms (eg, YouTube, Vine), and referral to traditional media (eg, website for the New England Journal of Medicine). We analyzed a total of 26 blogs (Multimedia Appendix 5) to explore the contextual meaning of the \#FOAMed community. Ethnographic examination showed forums of discussion by way of blog comments, with commenters also being members of the \#FOAMed Twitter community.

\section{Discussion}

\section{Principal Findings}

Analysis of the data revealed evidence of a community organized around a scale-free topology network built on the hashtag \#FOAMed, with an increasing number of users (nodes) and connections (links) manifested as impressions. The community is organized around members with a high degree of influence (centrality) that functions as a major hub for communication and knowledge management. In terms of content, knowledge appears to have a similar architecture, with relatively small pieces (blog posts or videos) concentrating most of the interactions and functioning also as hubs with high centrality $[32,33]$.

The outcomes of the \#FOAMed hashtag database analysis can be matched against the composite Aveling-Wenger $[6,12]$ postulate for a health care $\mathrm{CoP}$ (Table 1). Thus, there is preliminary evidence suggesting the \#FOAMed community possesses many of the characteristics of an oCoP and may contribute to recent literature describing learning and practice oCoPs [34].

In terms of the formation of interdependent groups and individuals (A1C) and the crossing of clinical and organizational boundaries (A2C), the data support the existence of a diverse group of people, organized around content and users hubs (Tables 1 and 2); the community includes health care providers in different roles (physicians, nurses, paramedics, etc), as well as health care and educational organizations; and there is robust interaction between users (nodes) of different background, trainings, and specialties. At the time of analysis, community members were mostly located in Western anglophone countries, with fewer members in Asia and Latin America (Multimedia Appendix 4). However, while it is possible to describe the volume of the dependencies, it is more challenging to demonstrate their meaning. It must be acknowledged that, due to the size of the community, it is difficult to completely verify the relationships and interactions that prove the interdependencies suggested by the Aveling-Wenger postulate. The analysis of an oCoP focuses on the members of the community and their interactions around pieces of the domain in order to construct a practice and is essentially different from a network analysis. This approach, although very relevant, would not have been appropriate for this work, given the lack of focus on content. Clearly, community members are engaged in the dissemination and sharing of medical knowledge, as demonstrated by the type and frequency of link sharing and the efforts of the community to increase the reach of the information through retweets (Multimedia Appendix 4). It is key to describe that all of the content analyzed was quite concentrated on medical knowledge, there were no links to nonbiomedical content, and the discussions were in a high degree quite focused on dissemination and critique; in other words, there was very minimal noise in the discussions among the members of the community (Multimedia Appendix 3). These actions embody the core of the common purpose postulate (A3D) and the exploitation of inherent networks (A5DCP). However, the nature of those networks is not clear and diffusion of knowledge cannot be encapsulated using this whole-system review approach, which is a limitation of a digital-based analysis of knowledge translation.

The most powerful characteristic of the \#FOAMed network [27] is the robustness and richness of the interactions between the users (nodes) through strong relations (links); this activity resulted in more than a billion relations (retweets or links) [30], with a clear delineation of network synergy (Figures 3 and 4; Table 4), which support achievement as well as learning and sharing knowledge (A4CDP) and vertical and lateral structure operation (A6C). The centrality maps show a classic scale-free topology, with clear hubs for content and users arranged in a mesh topology, illustrating a vertical and horizontal arrangement in the Aveling construct.

The measures of weight based on hub and authority showed evidence of peer influence and online social mechanisms that influence peers and community members and create subcommunities (Figures 3 and 4); this suggests social control mechanisms (postulate $\mathrm{A} 7 \mathrm{CP}$ ) and, combined with the activity related to link and knowledge, suggests evidence of harnessing the power and collective wisdom of the community (A8P) as shown in the netnographic analysis in Multimedia Appendix 3. This describes the top 150 conversation threads and demonstrates the branching structure, each with up to 15 different users and as many as 8 branches. A very small number of conversations with very few participants would have challenged the postulate that users all have a contribution to make. We acknowledge our analysis of the \#FOAMed community appears to show centralization around a few clusters of influence and not a completely distributed architecture. This appears to be related to coalescence of users around sources of knowledge and does imply that there are core users of the network as well as those at the periphery. 
The primary aim of this research was to show preliminary evidence of the emergence of an oCoP from the FOAM movement represented by Twitter interactions that included the \#FOAMed hashtag. The concept of CoP was introduced by Wenger [6] and describes the appearance of networks of people who interact explicitly, create and negotiate knowledge, and are able to translate this knowledge into a praxis. Our analysis indicates the possible emergence of a community, as evidenced by the large number of users, with a clear level of engagement and persistent participation across time. There was an exchange of information and ideas primarily through a microblogging format but nimbly cross-linking to platforms with more knowledge depth, such as blogs and media hubs (eg, YouTube and Vimeo). The community shows a topology similar to other mesh-organized networks, in particular high centrality hubs or users and content.

The main challenge of a digital community of people with little interaction in real life is translating the information, knowledge, and innovation into practice change [35]. However, the \#FOAMed community is able to generate content, refine its applicability, and identify tailored and meaningful adaptations based on the information negotiated. This is evidenced in the sentiment analysis of the content, as well as the organization around specific links. It appears that some members of the group serve as knowledge gatekeepers without forcing the community's focus (demonstrated by the significance of the links and by the concentrated domains where information is stored and appraised). In terms of evidence of real-world application of the knowledge created, analysis of the content of the information suggests they are actionable concepts; however, a proof of application in real practice is difficult to obtain using an online analysis and is a limitation of this study. The conversations held in the forum of the comments sections of the most commonly shared blogs showed the social construct of testing, challenging, and contextualizing new knowledge, and they challenged assumptions of the different clinical practice areas of the contributors. These interactions ranged from affirmations agreeing that the new knowledge was practice changing, to instances of reflection that changes had been made with positive results. We observed a process of affirmation, contextualization to current or changed practice, and presentation of further evidence by way of linking to other primary or secondary literature on the discussed topic.

\section{Limitations}

A major limitation of our study is that the fine detail interlinking community practice has not been confirmed. This will require a different methodological approach, which is not possible in the scope of this study. Given the clear matching of the dataset to the a priori postulate, further more detailed work seems worthwhile.

Further limitations to this work include the inability to confirm alterations in day-to-day patient clinical outcomes by network participants. However, the size of the network and its continued expansion and evolution suggest engagement through an evidence hierarchy that reaches beyond perceived or reported benefit. We believe the main implication of this preliminary evidence of an oCoP is the possible adoption of this structure (ie, a decentralized, distributed, self-regulated, diverse, nonhierarchical network of users and knowledge) as a suitable, relatively cheap and powerful method for knowledge management in health care teams and institutions.

The emergence of an oCoP oriented toward collaboration and the creation, curation, appraisal, and dissemination of knowledge would be a paradigm change in medical education. The concept of education based on a CoP have been described previously [11], but its existence with regard to medical education has never been shown before, although theoretical benefits have been considered and anxiously anticipated [9,36], with some authors arguing that social learning may replace formal training [9].

\section{Conclusion}

The advent of a network devoted to knowledge translation constitutes a new frontier for health care education and knowledge management, with consequences that we are just beginning to understand. Our work shows that social media have been innovative in progressing medical education and that identification of oCoPs within social media may well be possible. This new framework of knowledge organization may appear as a new model for the management of information and users in a network-based health care system.

\section{Acknowledgments}

We received an unrestricted grant from Symplur (California, USA) for the use of the Symplur Signals [37] analytic engine. The grant was awarded as part of a research challenge sponsored by the Stanford Medicine X conference [38]. The sponsors had no role in the review or approval of the manuscript for publication.

\section{Conflicts of Interest}

None declared.

\section{Multimedia Appendix 1}

Top 100 influencers in the \#FOAMed community. 


\section{Multimedia Appendix 2}

Weighted network centrality metrics, based on mentions, hub, and authority quotients.

[PDF File (Adobe PDF File), 25KB-Multimedia Appendix 2]

\section{Multimedia Appendix 3}

Top 150 conversations generated by the \#FOAMed community.

[PDF File (Adobe PDF File), 169KB-Multimedia Appendix 3]

\section{Multimedia Appendix 4}

Top 100 links shared by the \#FOAMed community.

[PDF File (Adobe PDF File), 26KB-Multimedia Appendix 4]

\section{Multimedia Appendix 5}

Netnographic evaluation of digital products from \#FOAMed conversations.

[PDF File (Adobe PDF File), 27KB-Multimedia Appendix 5]

\section{References}

1. Bazzoli GJ, Shortell SM, Dubbs N, Chan C, Kralovec P. A taxonomy of health networks and systems: bringing order out of chaos. Health Serv Res 1999 Feb;33(6):1683-1717 [FREE Full text] [Medline: 10029504]

2. Greenhalgh T, Wieringa S. Is it time to drop the 'knowledge translation' metaphor? A critical literature review. J R Soc Med 2011 Dec;104(12):501-509 [ [FREE Full text] [doi: 10.1258/jrsm.2011.110285] [Medline: 22179293]

3. Friesen N, Lowe S. The questionable promise of social media for education: connective learning and the commercial imperative. J Comput Assist Learn 2012 Jun;28(3):183-194. [doi: 10.1111/j.1365-2729.2011.00426.x]

4. Moran M, Seaman J, Tinti-Kane H. Teaching, learning, and sharing: how today's higher education faculty use social media. Boston, MA: Pearson Leaning Solutions and Babson Survey Research Group; 2011 Apr. URL: http://files.eric.ed.gov/ fulltext/ED535130.pdf [accessed 2017-06-28] [WebCite Cache ID 6rZ9RINya]

5. Dabbagh N, Kitsantas A. Personal learning environments, social media, and self-regulated learning: a natural formula for connecting formal and informal learning. Fairfax, VA: George Mason University URL: http://masononline.gmu.edu/ wp-content/uploads/Abstract N-Dabbagh.pdf [accessed 2017-02-28] [WebCite Cache ID 6ocR6nLGd]

6. Wenger E. Communities of practice and social learning systems: the career of a concept. URL: http://wenger-trayner.com/ wp-content/uploads/2012/01/09-10-27-CoPs-and-systems-v2.01.pdf [accessed 2017-02-28] [WebCite Cache ID 6ocSHq3A0]

7. Rheingold H. The Virtual Community: Homesteading on the Electronic Frontier. Cambridge, MA: MIT Press; 2000.

8. Dube L, Bourhis A, Jacob R. Towards a typology of virtual communities of practice. Interdisciplinary J Inf Knowledge Manage 2006;1:69-93 [FREE Full text]

9. Stamps D. Communities of practice: Learning is social, training is irrelevant? Training 1997 Feb;34(2):34-42.

10. Eisen MJ. Peer-based professional development viewed through the lens of transformative learning. Holist Nurs Pract 2001 Oct;16(1):30-42. [Medline: 15559045]

11. Li LC, Grimshaw JM, Nielsen C, Judd M, Coyte PC, Graham ID. Evolution of Wenger's concept of community of practice. Implement Sci 2009 Mar 01;4:11 [FREE Full text] [doi: 10.1186/1748-5908-4-11] [Medline: 19250556]

12. Aveling E, Martin G, Armstrong N, Banerjee J, Dixon-Woods M. Quality improvement through clinical communities: eight lessons for practice. J Health Organ Manag 2012;26(2):158-174. [doi: 10.1108/14777261211230754] [Medline: 22856174]

13. Lewis B, Rush D. Experience of developing Twitter-based communities of practice in higher education. Res Learning Technol 2013 Jun 11;21(1):18598. [doi: 10.3402/rlt.v21i0.18598]

14. Braun P. Digital knowledge networks: linking communities of practice with innovation. J Bus Strategies 2002;19(1):1-1 [FREE Full text]

15. Canadian Institutes of Health Research. Guide to knowledge translation planning at CIHR: integrated and end-of-grant approaches. 2012. URL: http://www.cihr-irsc.gc.ca/e/documents/kt lm ktplan-en.pdf [accessed 2017-06-28] [WebCite Cache ID 6rZBaDV6t]

16. Jalali A, Sherbino J, Frank J, Sutherland S. Social media and medical education: exploring the potential of Twitter as a learning tool. Int Rev Psychiatry 2015 Apr;27(2):140-146. [doi: 10.3109/09540261.2015.1015502] [Medline: 25768325]

17. Eysenbach G, Powell J, Englesakis M, Rizo C, Stern A. Health related virtual communities and electronic support groups: systematic review of the effects of online peer to peer interactions. BMJ 2004 May 15;328(7449):1166 [FREE Full text] [doi: 10.1136/bmj.328.7449.1166] [Medline: 15142921] 
18. Cadogan M, Thoma B, Chan TM, Lin M. Free Open Access Meducation (FOAM): the rise of emergency medicine and critical care blogs and podcasts (2002-2013). Emerg Med J 2014 Oct;31(e1):e76-e77. [doi: 10.1136/emermed-2013-203502] [Medline: 24554447]

19. Nickson CP, Cadogan MD. Free Open Access Medical education (FOAM) for the emergency physician. Emerg Med Australas 2014 Feb;26(1):76-83. [doi: 10.1111/1742-6723.12191] [Medline: 24495067]

20. Kozinets RV. The field behind the screen: using netnography for marketing research in online communities. J Marketing Res 2002 Feb;39(1):61-72. [doi: 10.1509/jmkr.39.1.61.18935]

21. Kozinets RV. Netnography: Doing Ethnographic Research Online. Thousand Oaks, CA: Sage Publications; 2009.

22. Boyd D, Crawford K. Critical questions for big data. Inf Commun Soc 2012 Mar 20;15(5):662-679.

23. Manovich L. www.manovich.net. 2011. Trending: the promises and the challenges of big social data URL: http://dhdebates. gc.cuny.edu/debates/text/15 [accessed 2015-12-23] [WebCite Cache ID 6ocRn5m92]

24. Singh K, John A. A study of tweet chats for breast cancer patients. New York, NY: ACM; 2015 Presented at: International Conference on Social Media \& Society; July 27-29, 2015; Toronto, ON, Canada p. 1-7.

25. Small TA. What the hashtag? Inf Commun Soc 2011 Sep;14(6):872-895. [doi: 10.1080/1369118X.2011.554572]

26. McPherson M, Smith-Lovin L, Cook JM. Birds of a feather: homophily in social networks. Annu Rev Sociol 2001 Aug;27(1):415-444. [doi: 10.1146/annurev.soc.27.1.415]

27. Wasserman S, Faust K. Social Network Analysis: Methods and Applications. Cambridge, UK: Cambridge University Press; 1994.

28. Kozinets R. Netnography: the marketer's secret weapon: how social media understanding drives innovation. Mountain View, CA: NetBase Solutions, Inc; 2010 Mar. URL: https://preventviolentextremism.info/sites/default/files/ White\%20Paper\%20\%E2\%80\%93\%20Netnography-\%20The\%20Marketer\%E2\%80\%99s\%20Secret\%20Weapon.pdf [accessed 2017-02-28] [WebCite Cache ID 6ocTArPsf]

29. Utengen A. Network analysis.: Symplur.com; 2017. URL: https://help.symplur.com/reports/people/network-analysis [accessed 2017-07-04] [WebCite Cache ID 6rhqMIKfT]

30. Kleinberg JM. Hubs, authorities, and communities. ACM Comput Surv 1999 Dec 1;31(4es):5. [doi: 10.1145/345966.345982]

31. Pang B, Lee L. Opinion mining and sentiment analysis. Found Trends Inf Retrieval 2008;2(1-2):1-135. [doi: $10.1561 / 1500000011]$

32. Myneni S, Cobb N, Cohen T. In pursuit of theoretical ground in behavior change support systems: analysis of peer-to-peer communication in a health-related online community. J Med Internet Res 2016 Feb 02;18(2):e28 [FREE Full text] [doi: 10.2196/jmir.4671] [Medline: 26839162]

33. Mishori R, Singh LO, Levy B, Newport C. Mapping physician Twitter networks: describing how they work as a first step in understanding connectivity, information flow, and message diffusion. J Med Internet Res 2014;16(4):e107 [FREE Full text] [doi: 10.2196/jmir.3006] [Medline: 24733146]

34. Ikioda F, Kendall S. Transformation of health visiting services in England using an online community of practice. Health Policy Technol 2016 Sep;5(3):298-306. [doi: 10.1016/j.hlpt.2016.02.006]

35. Cohen D, McDaniel RR, Crabtree BF, Ruhe MC, Weyer SM, Tallia A, et al. A practice change model for quality improvement in primary care practice. J Healthc Manag 2004;49(3):155-68; discussion 169. [Medline: 15190858]

36. Wilcock PM, Janes G, Chambers A. Health care improvement and continuing interprofessional education: continuing interprofessional development to improve patient outcomes. J Contin Educ Health Prof 2009;29(2):84-90. [doi:

10.1002/chp.20016] [Medline: 19530199]

37. Symplur signals.: Symplur LLC; 2017. URL: https://www.symplur.com/signals/ [accessed 2017-07-04] [WebCite Cache ID 6riFbf5I8]

38. Stanford Medicine $X$ and Symplur Signals. Stanford Medicine $X$ and Symplur announce social media analytics research challenge. URL: https://medicinex.stanford.edu/2015/02/13/medx-symplur-challenge/ [accessed 2017-07-04] [WebCite Cache ID 6rhrJoyil]

\section{Abbreviations}

CoP: community of practice

FOAM: free open access medical education

oCoP: online community of practice 
Edited by A Keepanasseril; submitted 29.11.16; peer-reviewed by G Petrič, S Aggarwal; comments to author 24.01.17; revised version received 06.03.17; accepted 14.06.17; published 14.07.17

Please cite as:

Roland D, Spurr J, Cabrera D

Preliminary Evidence for the Emergence of a Health Care Online Community of Practice: Using a Netnographic Framework for Twitter Hashtag Analytics

J Med Internet Res 2017;19(7):e252

URL: http://www.jmir.org/2017/7/e252/

doi: $10.2196 /$ jmir.7072

PMID: 28710054

CDamian Roland, Jesse Spurr, Daniel Cabrera. Originally published in the Journal of Medical Internet Research (http://www.jmir.org), 14.07.2017. This is an open-access article distributed under the terms of the Creative Commons Attribution License (https://creativecommons.org/licenses/by/4.0/), which permits unrestricted use, distribution, and reproduction in any medium, provided the original work, first published in the Journal of Medical Internet Research, is properly cited. The complete bibliographic information, a link to the original publication on http://www.jmir.org/, as well as this copyright and license information must be included. 